OPEN $\curvearrowright$ ACCESS

드 COMMUNICATIONS

ISSN 2056-9890

\section{Crystal structure of (E)-1-(3-chlorophen- yl)-3-(furan-2-yl)prop-2-en-1-one}

Sarah K. Zingales, ${ }^{*}$ Maya Z. Wallace and Clifford W. Padgett

Armstrong State University, Department of Chemistry and Physics, 11935 Abercorn St., Savannah, GA 31419, USA. *Correspondence e-mail:

sarah.zingales@armstrong.edu

Received 20 August 2015; accepted 31 August 2015

Edited by H. Stoeckli-Evans, University of Neuchâtel, Switzerland

The title compound, $\mathrm{C}_{13} \mathrm{H}_{9} \mathrm{ClO}_{2}$, exhibits a non-planar geometry; the furan ring being inclined to the benzene ring by $50.52(16)^{\circ}$. In the crystal, molecules stack along the $a$ axis; however, there are no significant intermolecular interactions present.

Keywords: crystal structure; chalcone.

CCDC reference: 1421581

\section{Related literature}

For the synthesis of the title compound, see: HanLee et al. (2011). For the syntheses of related compounds, see: Inokuma et al. (2009).<smiles>O=C(/C=C/c1ccoc1)c1cccc(Cl)c1</smiles>

\section{Experimental}

\subsection{Crystal data}

$\mathrm{C}_{13} \mathrm{H}_{9} \mathrm{ClO}_{2}$

$M_{r}=232.67$

Monoclinic, $P 2_{1} / c$

$a=7.186(8) \AA$

$b=25.77(3) \AA$

$c=5.774(6) \AA$

$\beta=94.734(10)^{\circ}$

$$
\begin{aligned}
& V=1066(2) \AA^{3} \\
& Z=4 \\
& \text { Mo } K \alpha \text { radiation } \\
& \mu=0.34 \mathrm{~mm}^{-1} \\
& T=173 \mathrm{~K} \\
& 0.30 \times 0.30 \times 0.10 \mathrm{~mm}
\end{aligned}
$$

\subsection{Data collection}

Rigaku XtaLAB mini diffractometer

Absorption correction: multi-scan (REQAB; Rigaku, 1998)

$T_{\min }=0.832, T_{\max }=0.967$

11328 measured reflections 2461 independent reflections 1672 reflections with $F^{2}>2.0 \sigma\left(F^{2}\right)$ $R_{\text {int }}=0.080$

\subsection{Refinement}

$R\left[F^{2}>2 \sigma\left(F^{2}\right)\right]=0.054$

$w R\left(F^{2}\right)=0.128$

$S=1.07$

2461 reflections

145 parameters

$\mathrm{H}$-atom parameters constrained

$\Delta \rho_{\max }=0.29{\mathrm{e} \AA^{-3}}^{-3}$

$\Delta \rho_{\min }=-0.33 \mathrm{e}^{-3}$

Data collection: CrystalClear-SM Expert (Rigaku, 2011); cell refinement: CrystalClear-SM Expert; data reduction: CrystalClear-SM Expert; program(s) used to solve structure: SHELXS97 (Sheldrick, 2008); program(s) used to refine structure: SHELXL97 (Sheldrick, 2008); molecular graphics: CrystalStructure (Rigaku, 2011); software used to prepare material for publication: CrystalStructure.

\section{Acknowledgements}

MZW is grateful for the financial support of an ASU Undergraduate Research Grant and SKZ for an ASU Research and Scholarship Grant.

Supporting information for this paper is available from the IUCr electronic archives (Reference: SU5196).

\section{References}

HanLee, I.-S., Jeon, H.-J. \& Lee, C.-K. (2011). Bull. Korean Chem. Soc. 32, 687-692.

Rigaku (1998). REQAB. Rigaku Corporation, Tokyo, Japan.

Rigaku (2011). CrystalClear-SM Expert and CrystalStructure. Rigaku Corporation, Tokyo, Japan.

Sheldrick, G. M. (2008). Acta Cryst. A64, 112-122.

Inokuma, T., Nagamoto, Y., Sakamoto, S., Miyabe, H., Takasu, K. \& Takemoto, Y. (2009). Heterocycles, 79, 573-582. 


\section{supporting information}

Acta Cryst. (2015). E71, o707 [doi:10.1107/S2056989015016266]

\section{Crystal structure of $(E)$-1-(3-chlorophenyl)-3-(furan-2-yl)prop-2-en-1-one}

\section{Sarah K. Zingales, Maya Z. Wallace and Clifford W. Padgett}

\section{S1. Chemical context}

The title compound is a chalcone analog that is currently being studied for its potential anticancer activity [unpublished results]. Its synthesis and NMR characteristics have been reported (HanLee et al. 2011), but its crystal structure has not been reported until now.

\section{S2. Synthesis and crystallization}

The title compound was synthesized by an aldol condensation reaction. 3-furaldehyde $(1 \mathrm{mmol})$ and 3-chloroacetophenone $(1 \mathrm{mmol})$ were dissolved in ethanol $(5 \mathrm{ml})$. A NaOH solution $(5 \mathrm{M}, 1 \mathrm{~mL})$ was added and the reaction mixture was stirred until a precipitate formed. The reaction mixture was cooled in an ice bath for $20 \mathrm{~min}$. The solids formed were filtered off and recrystallized from $\mathrm{MeOH} / \mathrm{H}_{2} \mathrm{O}(1: 1, \mathrm{v}: \mathrm{v})$. Slow evaporation of a solution of the title compound in $\mathrm{MeOH}$ gave pale brown crystals.

\section{S3. Refinement}

Crystal data, data collection and structure refinement details are summarized in Table 1. The $\mathrm{H}$ atoms were included in calculated positions and treated as riding atoms: $\mathrm{C}-\mathrm{H}=0.95 \AA$ with $\mathrm{U}_{\mathrm{iso}}(\mathrm{H})=1.2 \mathrm{U}_{\mathrm{eq}}(\mathrm{C})$.

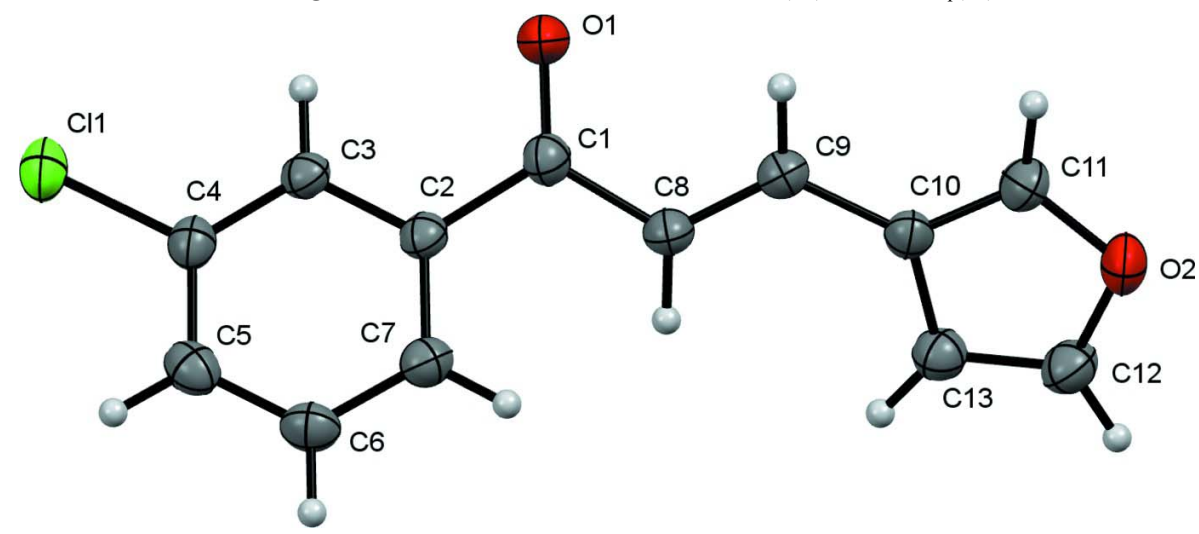

\section{Figure 1}

A view of the molecular structure of the title compound, with atom labelling. Displacement ellipsoids are drawn at the $50 \%$ probability level.

\section{(E)-1-(3-Chlorophenyl)-3-(furan-2-yl)prop-2-en-1-one}

Crystal data

$\mathrm{C}_{13} \mathrm{H}_{9} \mathrm{ClO}_{2}$

$M_{r}=232.67$

Monoclinic, $P 2{ }_{1} / c$

Hall symbol: -P $2 \mathrm{ybc}$ 
$a=7.186(8) \AA$

$b=25.77$ (3) $\AA$

$c=5.774(6) \AA$

$\beta=94.734(10)^{\circ}$

$V=1066(2) \AA^{3}$

$Z=4$

$F(000)=480.00$

$D_{\mathrm{x}}=1.450 \mathrm{Mg} \mathrm{m}^{-3}$

Data collection

Rigaku XtaLAB mini diffractometer

Detector resolution: 6.827 pixels $\mathrm{mm}^{-1}$

$\omega$ scans

Absorption correction: multi-scan

(REQAB; Rigaku, 1998)

$T_{\min }=0.832, T_{\max }=0.967$

11328 measured reflections

Refinement

Refinement on $F^{2}$

$R\left[F^{2}>2 \sigma\left(F^{2}\right)\right]=0.054$

$w R\left(F^{2}\right)=0.128$

$S=1.07$

2461 reflections

145 parameters

0 restraints

Primary atom site location: structure-invariant direct methods
Mo $K \alpha$ radiation, $\lambda=0.71075 \AA$

Cell parameters from 2235 reflections

$\theta=2.4-27.5^{\circ}$

$\mu=0.34 \mathrm{~mm}^{-1}$

$T=173 \mathrm{~K}$

Prism, colorless

$0.30 \times 0.30 \times 0.10 \mathrm{~mm}$

2461 independent reflections

1672 reflections with $F^{2}>2.0 \sigma\left(F^{2}\right)$

$R_{\text {int }}=0.080$

$\theta_{\max }=27.6^{\circ}$

$h=-9 \rightarrow 9$

$k=-33 \rightarrow 33$

$l=-7 \rightarrow 7$

Secondary atom site location: difference Fourier map

Hydrogen site location: inferred from neighbouring sites

$\mathrm{H}$-atom parameters constrained

$w=1 /\left[\sigma^{2}\left(F_{\mathrm{o}}^{2}\right)+(0.0293 P)^{2}+0.6811 P\right]$ where $P=\left(F_{\mathrm{o}}{ }^{2}+2 F_{\mathrm{c}}{ }^{2}\right) / 3$

$(\Delta / \sigma)_{\max }<0.001$

$\Delta \rho_{\max }=0.29 \mathrm{e} \AA^{-3}$

$\Delta \rho_{\min }=-0.33$ e $\AA^{-3}$

Special details

Refinement. Refinement was performed using all reflections. The weighted $R$-factor $(w R)$ and goodness of fit $(S)$ are based on $F^{2} . R$-factor (gt) are based on $F$. The threshold expression of $F^{2}>2.0 \sigma\left(F^{2}\right)$ is used only for calculating $R$-factor (gt).

Fractional atomic coordinates and isotropic or equivalent isotropic displacement parameters $\left(\AA^{2}\right)$

\begin{tabular}{lllll}
\hline & $x$ & $y$ & $z$ & $U_{\text {iso }} * / U_{\text {eq }}$ \\
\hline C11 & $0.25413(11)$ & $0.29816(3)$ & $0.35320(13)$ & $0.0404(3)$ \\
O1 & $0.2759(3)$ & $0.50459(7)$ & $0.3133(4)$ & $0.0385(6)$ \\
O2 & $0.3111(3)$ & $0.71261(7)$ & $0.9248(4)$ & $0.0395(5)$ \\
C1 & $0.2650(4)$ & $0.49620(10)$ & $0.5220(5)$ & $0.0295(6)$ \\
C2 & $0.2198(4)$ & $0.44238(10)$ & $0.6022(5)$ & $0.0263(6)$ \\
C3 & $0.2537(4)$ & $0.40038(10)$ & $0.4594(5)$ & $0.0271(6)$ \\
C4 & $0.2074(4)$ & $0.35117(10)$ & $0.5274(5)$ & $0.0289(6)$ \\
C5 & $0.1236(4)$ & $0.34244(11)$ & $0.7323(5)$ & $0.0335(7)$ \\
C6 & $0.0897(4)$ & $0.38439(11)$ & $0.8733(5)$ & $0.0334(7)$ \\
C7 & $0.1396(4)$ & $0.43435(11)$ & $0.8108(5)$ & $0.0304(7)$ \\
C8 & $0.2914(4)$ & $0.53727(10)$ & $0.6976(5)$ & $0.0293(6)$ \\
C9 & $0.2727(4)$ & $0.58734(10)$ & $0.6361(5)$ & $0.0271(6)$ \\
C10 & $0.3055(4)$ & $0.63097(10)$ & $0.7933(5)$ & $0.0275(6)$ \\
C11 & $0.2624(4)$ & $0.68115(11)$ & $0.7414(5)$ & $0.0338(7)$
\end{tabular}




\begin{tabular}{lllll}
$\mathrm{C} 12$ & $0.3882(5)$ & $0.68125(12)$ & $1.0974(5)$ & $0.0369(7)$ \\
$\mathrm{C} 13$ & $0.3873(4)$ & $0.63144(11)$ & $1.0275(5)$ & $0.0327(7)$ \\
$\mathrm{H} 3$ & 0.3082 & 0.4057 & 0.3168 & $0.0325^{*}$ \\
$\mathrm{H} 5$ & 0.0902 & 0.3083 & 0.7751 & $0.0402^{*}$ \\
$\mathrm{H} 6$ & 0.0320 & 0.3790 & 1.0135 & $0.0400^{*}$ \\
$\mathrm{H} 7$ & 0.1190 & 0.4628 & 0.9100 & $0.0365^{*}$ \\
$\mathrm{H} 8$ & 0.3220 & 0.5284 & 0.8559 & $0.0352^{*}$ \\
$\mathrm{H} 9$ & 0.2353 & 0.5948 & 0.4780 & $0.0325^{*}$ \\
$\mathrm{H} 11$ & 0.2058 & 0.6927 & 0.5960 & $0.0406^{*}$ \\
$\mathrm{H} 12$ & 0.4359 & 0.6930 & 1.2466 & $0.0443^{*}$ \\
$\mathrm{H} 13$ & 0.4323 & 0.6023 & 1.1162 & $0.0393^{*}$ \\
\hline
\end{tabular}

Atomic displacement parameters $\left(\AA^{2}\right)$

\begin{tabular}{lllllll}
\hline & $U^{11}$ & $U^{22}$ & $U^{33}$ & $U^{12}$ & $U^{13}$ & $U^{23}$ \\
\hline C11 & $0.0503(5)$ & $0.0258(4)$ & $0.0454(5)$ & $0.0004(4)$ & $0.0067(4)$ & $-0.0059(3)$ \\
O1 & $0.0601(15)$ & $0.0292(11)$ & $0.0266(11)$ & $0.0010(10)$ & $0.0055(10)$ & $0.0005(9)$ \\
O2 & $0.0520(13)$ & $0.0243(11)$ & $0.0418(12)$ & $-0.0012(10)$ & $0.0011(10)$ & $-0.0057(9)$ \\
C1 & $0.0321(15)$ & $0.0254(14)$ & $0.0308(15)$ & $0.0027(12)$ & $0.0017(12)$ & $-0.0006(12)$ \\
C2 & $0.0285(14)$ & $0.0268(14)$ & $0.0231(13)$ & $0.0008(12)$ & $-0.0017(11)$ & $-0.0017(11)$ \\
C3 & $0.0289(15)$ & $0.0297(15)$ & $0.0224(14)$ & $-0.0024(12)$ & $0.0002(11)$ & $-0.0026(11)$ \\
C4 & $0.0294(15)$ & $0.0258(14)$ & $0.0308(15)$ & $0.0019(12)$ & $-0.0023(12)$ & $-0.0024(12)$ \\
C5 & $0.0319(16)$ & $0.0331(16)$ & $0.0347(16)$ & $-0.0032(13)$ & $-0.0026(13)$ & $0.0051(12)$ \\
C6 & $0.0314(16)$ & $0.0391(17)$ & $0.0302(15)$ & $-0.0022(13)$ & $0.0065(12)$ & $0.0035(13)$ \\
C7 & $0.0311(15)$ & $0.0339(16)$ & $0.0262(14)$ & $0.0024(13)$ & $0.0023(12)$ & $-0.0014(12)$ \\
C8 & $0.0351(16)$ & $0.0285(14)$ & $0.0239(14)$ & $0.0001(13)$ & $-0.0001(11)$ & $0.0000(11)$ \\
C9 & $0.0272(14)$ & $0.0294(14)$ & $0.0247(14)$ & $0.0002(12)$ & $0.0022(11)$ & $-0.0014(11)$ \\
C10 & $0.0287(14)$ & $0.0271(14)$ & $0.0275(14)$ & $-0.0018(12)$ & $0.0062(11)$ & $-0.0008(11)$ \\
C11 & $0.0397(17)$ & $0.0307(15)$ & $0.0304(15)$ & $-0.0047(13)$ & $-0.0009(13)$ & $-0.0035(12)$ \\
C12 & $0.0419(18)$ & $0.0388(17)$ & $0.0295(15)$ & $0.0016(15)$ & $-0.0007(13)$ & $-0.0049(13)$ \\
C13 & $0.0338(16)$ & $0.0335(16)$ & $0.0307(15)$ & $0.0020(14)$ & $0.0008(12)$ & $0.0002(12)$ \\
& & & & & &
\end{tabular}

Geometric parameters $\left(A,{ }^{\circ}\right)$

\begin{tabular}{llll}
\hline $\mathrm{C} 11-\mathrm{C} 4$ & $1.746(3)$ & $\mathrm{C} 4-\mathrm{C} 5$ & $1.389(5)$ \\
$\mathrm{O} 1-\mathrm{C} 1$ & $1.233(4)$ & $\mathrm{C} 5-\mathrm{C} 6$ & $1.387(5)$ \\
$\mathrm{O} 2-\mathrm{C} 11$ & $1.357(4)$ & $\mathrm{C} 6-\mathrm{C} 7$ & $1.392(5)$ \\
$\mathrm{O} 2-\mathrm{C} 12$ & $1.365(4)$ & $\mathrm{C} 8-\mathrm{C} 9$ & $1.342(4)$ \\
$\mathrm{C} 1-\mathrm{C} 2$ & $1.506(4)$ & $\mathrm{C} 9-\mathrm{C} 10$ & $1.452(4)$ \\
$\mathrm{C} 1-\mathrm{C} 8$ & $1.467(4)$ & $\mathrm{C} 10-\mathrm{C} 11$ & $1.357(4)$ \\
$\mathrm{C} 2-\mathrm{C} 3$ & $1.394(4)$ & $\mathrm{C} 10-\mathrm{C} 13$ & $1.429(4)$ \\
$\mathrm{C} 2-\mathrm{C} 7$ & $1.393(4)$ & $\mathrm{C} 12-\mathrm{C} 13$ & $1.346(5)$ \\
$\mathrm{C} 3-\mathrm{C} 4$ & $1.377(4)$ & & \\
$\mathrm{C} 11-\mathrm{O} 2-\mathrm{C} 12$ & & & $118.9(3)$ \\
$\mathrm{O} 1-\mathrm{C} 1-\mathrm{C} 2$ & $106.2(3)$ & $\mathrm{C} 4-\mathrm{C} 5-\mathrm{C} 6$ & $120.5(3)$ \\
$\mathrm{O} 1-\mathrm{C} 1-\mathrm{C} 8$ & $119.7(3)$ & $\mathrm{C} 5-\mathrm{C} 6-\mathrm{C} 7$ & $119.7(3)$ \\
$\mathrm{C} 2-\mathrm{C} 1-\mathrm{C} 8$ & $122.3(3)$ & $\mathrm{C} 2-\mathrm{C} 7-\mathrm{C} 6$ & $120.4(3)$
\end{tabular}




$\begin{array}{llll}\mathrm{C} 1-\mathrm{C} 2-\mathrm{C} 3 & 118.7(3) & \mathrm{C} 8-\mathrm{C} 9-\mathrm{C} 10 & 124.9(3) \\ \mathrm{C} 1-\mathrm{C} 2-\mathrm{C} 7 & 121.2(3) & \mathrm{C} 9-\mathrm{C} 10-\mathrm{C} 11 & 125.3(3) \\ \mathrm{C} 3-\mathrm{C} 2-\mathrm{C} 7 & 120.1(3) & \mathrm{C} 9-\mathrm{C} 10-\mathrm{C} 13 & 129.1(3) \\ \mathrm{C} 2-\mathrm{C} 3-\mathrm{C} 4 & 119.2(3) & \mathrm{C} 11-\mathrm{C} 10-\mathrm{C} 13 & 105.6(3) \\ \mathrm{C} 11-\mathrm{C} 4-\mathrm{C} 3 & 119.6(3) & \mathrm{O} 2-\mathrm{C} 11-\mathrm{C} 10 & 110.9(3) \\ \mathrm{C} 11-\mathrm{C} 4-\mathrm{C} 5 & 118.8(3) & \mathrm{O} 2-\mathrm{C} 12-\mathrm{C} 13 & 110.7(3) \\ \mathrm{C} 3-\mathrm{C} 4-\mathrm{C} 5 & 121.6(3) & \mathrm{C} 10-\mathrm{C} 13-\mathrm{C} 12 & 106.5(3)\end{array}$

